OPEN ACCESS

Edited by:

Hong Xu,

Nanyang Technological

University, Singapore

Reviewed by:

Supriya Ray,

Allahabad University, India

Kerstin Fröber,

University of Regensburg, Germany

${ }^{*}$ Correspondence:

Jinhong Ding

dingjh@cnu.edu.cn

Specialty section:

This article was submitted to

Cognition,

a section of the journal

Frontiers in Psychology

Received: 20 July 2021 Accepted: 15 September 2021 Published: 15 October 2021

Citation:

Zhou Q, Jiang Z and Ding J (2021)

Reward Expectation Differentially Modulates Global and Local Spatial

Working Memory Accuracy.

Front. Psychol. 12:744400.

doi: 10.3389/fpsyg.2021.744400

\section{Reward Expectation Differentially Modulates Global and Local Spatial Working Memory Accuracy}

\author{
Qingjie Zhou, Zanzan Jiang and Jinhong Ding* \\ Beijing Key Laboratory of Learning and Cognition, College of Psychology, Capital Normal University, Beijing, China
}

Although it has been suggested that reward expectation affects the performance of spatial working memory tasks, controversial results have been found in previous experiments. Hence, it is still unclear to what extent reward expectation has an effect on working memory. To clarify this question, a memory-guided saccade task was applied, in which participants were instructed to retain and reconstruct a temporospatial sequence of four locations by moving their eyes in each trial. The global- and local-level spatial working memory accuracies were calculated to determine the reward effect on the global and local level of processing in spatial working memory tasks. Although high reward expectation enhanced the encoding of spatial information, the percentage of trials in which the cued location was correctly fixated decreased with increment of reward expectation. The reconstruction of the global temporospatial sequence was enhanced by reward expectation, whereas the local reconstruction performance was not affected by reward. Furthermore, the improvements in local representations of uncued locations and local sequences were at the cost of the representation of cued locations. The results suggest that the reward effect on spatial working memory is modulated by the level of processing, which supports the flexible resource theory during maintenance.

Keywords: reward expectation, spatial working memory, global-level processing, local-level processing, high cognitive load

\section{INTRODUCTION}

Working memory plays an essential role in human adaptive behavior and lies at the core of cognitive psychology research since its birth. The limited capacity of working memory has been an important concern since the insightful research of Miller on "the magical number seven" (Miller, 1956), and it has been demonstrated that the capacity of working memory is even less than seven (Cowan, 2001; Kawasaki and Yamaguchi, 2013; Marchette et al., 2015). However, the total capacity of working memory is not fixed; it varies across individuals (Just and Carpenter, 1992; Barrett et al., 2004) and can even be improved by motivation within the same individual (Krawczyk et al., 2007; Heitz et al., 2008; Kawasaki and Yamaguchi, 2013).

As an extrinsic motivation, monetary reward is a powerful modulator of attention. The expectation of a monetary reward narrows down the scope of attention (Filetti et al., 2019), allocates cognitive resources (Wei and Kang, 2014; Su et al., 2021), and alters cognitive executive function (Qu et al., 2013). It has been proposed that reward expectation improves working memory capacity by encouraging participants to make more efforts to fulfill the working memory tasks (Gilbert and Fiez, 2004; Hopstaken et al., 2016). 
However, empirical results have revealed a complex relationship between reward and working memory. Under some circumstances, reward did improve working memory performance (Gilbert and Fiez, 2004; Taylor et al., 2004; Rowe et al., 2008; Beck et al., 2010; Savine et al., 2010; Marquand et al., 2011; Kawasaki and Yamaguchi, 2013; Sandry et al., 2014; Choi et al., 2015; Hammer et al., 2015; Fairclough and Ewing, 2017; Hefer and Dreisbach, 2017; Heritage et al., 2017; Klink et al., 2017; Allen and Ueno, 2018; Anna and Anna, 2018; Thurm et al., 2018; Gaillard et al., 2019; Magis-Weinberg et al., 2019; Manga et al., 2020; Sandry and Ricker, 2020), while other studies did not find the reward effect on working memory accuracy (Pochon et al., 2002; Krawczyk et al., 2007; Beck et al., 2010; Hager et al., 2015; Infanti et al., 2017; Smith et al., 2017; Fairclough et al., 2018; Di Rosa et al., 2019). A potential factor mediating the reward effect is working memory load. For example, the reward effect was pronounced in studies of complex tasks, such as remembering 20 locations (Cho et al., 2018) or maintaining spatial and other features simultaneously (Rowe et al., 2008; Kawasaki and Yamaguchi, 2013; Klink et al., 2017; Allen and Ueno, 2018; Anna and Anna, 2018; Gaillard et al., 2019). In contrast, when the task was relatively simple, such as the eight-arm maze task (Smith et al., 2017), the influence of reward expectation on spatial working memory disappeared. Some researchers directly manipulated working memory load and/or compared performances of participants with different working memory abilities (Taylor et al., 2004; Esteban et al., 2015; Hammer et al., 2015; Thurm et al., 2018; Gaillard et al., 2019). For example, Hammer et al. (2015) required children with attention-deficit/hyperactivity disorder (ADHD) and normally developed children to complete 2-back visual working memory tasks. The behavioral results revealed the reward expectation effect on working memory performance only in the ADHD group but not in the normally developed group. It was proposed that the disappearance of the reward effect was due to the ceiling effect (Savine et al., 2010; Esteban et al., 2015; Hammer et al., 2015).

However, the reward effect is not merely mediated by working memory load (Pochon et al., 2002; Heritage et al., 2017; Fairclough et al., 2018; Gaillard et al., 2019), and participants with better working memory ability have shown the reward effect, while others did not (Thurm et al., 2018; Manga et al., 2020). Hence, working memory load is not sufficient to explain the inconsistent results of the previous studies. There are other factors involved in the relationship between reward and working memory, such as processing level (Ahmed and Fockert, 2012) and other factors (Beck et al., 2010; Savine et al., 2010; Choi et al., 2015; Hammer et al., 2015; Heritage et al., 2017; Fairclough et al., 2018; Magis-Weinberg et al., 2019).

According to the load theory, the effectiveness of voluntary attention is impaired when working memory load is high, because of exhausting cognitive resources (Lavie et al., 2004). However, according to Ahmed and Fockert (2012), this theory is valid only when the task requires relative local-level processing, while the effect reverses when global-level processing is required. Ahmed and Fockert (2012) suggested that the ability to effectively concentrate attention to relevant local visual fields is reduced when working memory load is high. In typical spatial working memory studies, increment of spatial working memory load always couples with a more localized requirement of processing. Compared with low spatial working memory load condition, the visual field in high working memory load condition is divided into relatively small areas, and attention must be constrained to a more local visual field to take in distinct representations of multiple locations (Saarinen, 1988), while the effectiveness of voluntary attention deteriorates when the working memory load is high. Thus, the increase in working memory requirement is not only a burden to the maintenance of spatial information but also weakens the encoding of spatial locations.

The interaction between the effect of reward and the effect of working memory load may reflect the unstable reward effect on working memory. The reward effect improves working memory performance by enhancing voluntary attention (Gilbert and Fiez, 2004; Pessoa, 2009), but a high working memory load undermines the precision of spatial representations (Ahmed and Fockert, 2012). Based on these findings, we hypothesized that reward enhances spatial working memory performance at the global level, but working memory performance at the local level benefits less from reward or, even worse, the reward effect vanishes.

To verify this hypothesis, we applied a sequential memoryguided saccade task under different reward conditions. Compared with other delay-match tasks, the memory-guided saccade task is more flexible and accountable (Funahashi et al., 1993) and has been widely applied in studies of spatial working memory (Funahashi et al., 1993; Park et al., 1995; Sawaguchi and Iba, 2001; Johnston and Everling, 2008; Tsujimoto and Postle, 2012). The accuracy of saccade relies on precise spatial representation (Vergilino and Beauvillain, 2001; Theeuwes et al., 2009). The memory of sequential locations consists of location and sequence information. Sequential information is organized in either time or space, which are compatible (Fischer-Baum and Benjamin, 2014). Serial-order memory is highly connected with spatial working memory, and sequence information is represented in the form of space (van Dijck et al., 2013; Antoine et al., 2018).

In this study, spatial working memory performance was assessed both at the global and local level of similarity between stimuli sequence and scan path. In addition to the saccade landing point, fixation duration was calculated as a measurement of cognitive effort devoted to the task. Eye movements reflect the status of attention (Rayner, 1978, 2009; Theeuwes et al., 2009), and fixation duration is a valid indicator of attention (Rayner et al., 2007; Papageorgiou et al., 2014). Friedman and Liebelt (1981) found that fixation duration was prolonged when the gazed object was unusual or was required to be remembered. They suggested that the prolonged fixation duration reflects additional attention allocated to the gazed object. Considering that fixation duration covers multiple cognitive procedures, such as intake of foveal information and saccade planning (Rayner, 1998; Ludwig et al., 2014), and is influenced by factors other than cognitive effort (Rayner, 1998; Ludwig et al., 2014), comparing the fixation durations of reward cue and non-cue can provide 
more details about the impact of reward expectation on working memory.

\section{METHODS}

\section{Participants}

Twenty right-handed college students (10 men and 10 women) participated in this experiment. All of them had a normal or correct-to-normal vision and color vision. They were compensated after the experiment with a basic amount of money plus a bonus depending on their performance.

\section{Equipment and Materials}

Stimuli were presented on a 19-inch CRT monitor with a refresh rate of $120 \mathrm{~Hz}$ and a resolution of $1,024 \times 768$ pixels via Visual Basic. The viewing distance was $60 \mathrm{~cm}$. A chin and forehead rest was used to reduce head movements. Eye positions were recorded by SMI Hi-speed eye tracker (SensoMotoric Instruments $\mathrm{GmbH}$, Teltow, Germany) at a sampling rate of $350 \mathrm{~Hz}$. The spatial resolution of the eye tracker was $0.1^{\circ}$ of visual angle. A standard nine-point calibration and validation were conducted at the beginning of each block to ensure the eye data quality. Memory arrays consisted of four items, three of which were gray disks, while the fourth, a reward cue, was either a 1-Fen or 1-Yuan or blurred Chinese coin (1 Yuan equals 100 Fen in Chinese currency). The locations of reward cues in memory arrays were randomized. All stimuli were in the size of $5.59^{\circ}$ visual angle and had the same luminance (Figure 1B). The reward expectation level was assigned to none, low, and high corresponding to reward cues of blurred, 1-Fen, and 1-Yuan Chinese coins (reward cues), respectively.

The screen was divided into 16 grids and equally distributed in four quadrants (four squares in each quadrant). Items (disks or reward cues) were displayed in squares in accordance with the experimental design.

\section{Procedure}

A sample test paradigm was used in the experiment. During the encoding phase, a "+" appeared at the center of the screen and disappeared until the subjects fixated it steadily for at least $800 \mathrm{~ms}$. Four pictures, including three gray disks and a reward cue (blurred/1-Fen/1-Yuan Chinese coins), were presented in each quadrant at one of four grids for $1,000 \mathrm{~ms}$, sequentially. The stimulus positions were described by the sequential order of quadrants (denoted by bigger numbers) and the number of grids (labeled by letters; as shown in Figure 1A) in which the stimuli were displayed. The participants were asked to gaze at the pictures and to remember their locations and sequential order.

There was a delay of $800 \mathrm{~ms}$ before the recall phase. During the recall phase, the participants were instructed to reconstruct the spatial location sequence as precisely as possible in 5,000 ms by gazing on a blank screen. The eye scan path was described by two series of positions of different processing levels. One was the quadrant sequence, indicating the sequential order of eye positions in different quadrants, such as "1234" (denoted by letters in Figure 1A). The other one was the sequential positions at grids in each of the quadrants, denoted by letters "ABCD." The recall performance of spatial working memory was calculated by comparing the sequences of stimulus in the encoding phase and eye scan path in the retrieval phase. Similarities of each trial were calculated according to Brandt and Stark (1997) and Eddy (2004), including global similarity (GS), which was based on the quadrant, and local similarity (LS), which was based on the grid. Both GS and LS refer to degrees of similarity between sequences of stimuli positions in the study phase and eye fixation positions in the recall phase, ranging from 0 (totally different) to 1 (the same). GS and LS indicate the recall accuracy of the global and local positions of stimuli, respectively. LS has a finer spatial scale than GS and reflects a more rigid requirement of spatial resolution.

At the end of each trial, a feedback of reward amount (money in Chinese Yuan) was shown for 1,000 ms. Coin pictures were used to indicate locations to be remembered and reward cues (Hager et al., 2015; Di Rosa et al., 2019; Manga et al., 2020). The amount of reward depended on the performance (i.e., GS and LS) and reward conditions of participants and was calculated by the formula $\left.W^{*}(\mathrm{GS}+\mathrm{LS}) / 2\right)$, where $W=0$ for No-reward, $W=1$ for Low-reward, and $W=10$ for High-reward. The unit of it is the Chinese Yuan.

A total of 192 trials in the whole experiment were randomized and assigned into 12 blocks. There were 48 trials in each of the reward expectation conditions (No, Low, and High). The procedure of one trial is shown in Figure 2.

\section{Data Preprocessing}

Dependent variables in the present research were derived from the fixation data. Fixations were defined using a temporal threshold of $100 \mathrm{~ms}$ and a spatial threshold of $2^{\circ}$ visual angle, which were calculated offline. Then, the mean fixation number and the mean fixation duration were generated and grouped by the reward expectation conditions and location of fixation. The percentage of trials in which the cued grid was correctly refixed was used as the index of reward-cue memory performance. The spatial working memory task performance was evaluated by the sequence similarity proposed by Eddy (2004). Specifically, locations with the same temporal order of stimuli in the study phase and of eye fixation in the recall phase were compared, and each pair of overlapped locations scored 1 point. Sequence similarity was calculated by dividing the total score of four pairs of locations by 4 , with a range from 0 (totally wrong) to 1 (totally correct).

\section{RESULTS}

\section{Eye Fixations During the Study Phase}

During the study phase, three gray disks and a reward cue were presented sequentially one by one. The subjects were instructed to remember their spatial and temporal locations. We segregated the numbers of eye fixations and their durations by whether they were on the cue or non-cue (gray disk; as shown in Figure 3).

There was no significant effect of reward expectation or fixation position (on non-cue or cue) on the number of fixations. However, the fixation duration was significantly affected by reward expectation $\left[M_{\text {No-reward }}=(362 \pm 14) \mathrm{ms} ; M_{\text {Low-reward }}=\right.$ 


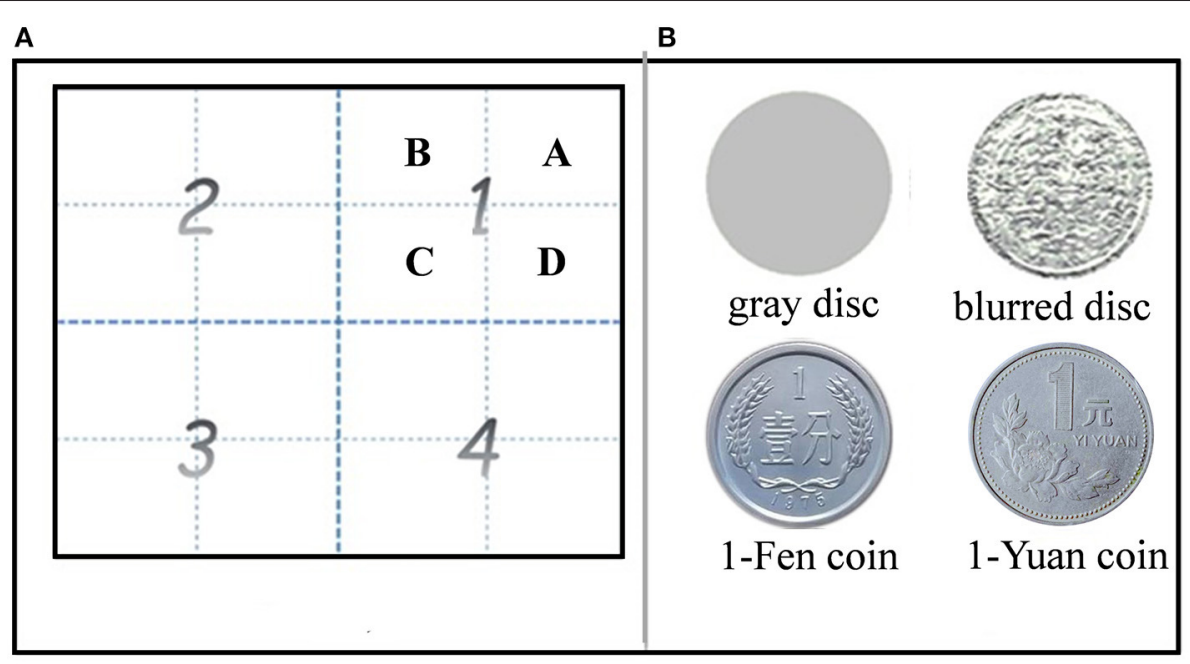

FIGURE 1 | Partition of the screen for stimulus presentation (A) and stimuli used in the experiment (B). (A) Four quadrants (marked by numbers) were divided into a total of 16 equal-size grids (four per quadrant, labeled by letters). (B) Stimuli were gray disk (top left), blurred disk (top right), 1-Fen (bottom left), and 1-Yuan (bottom right) Chinese coins.

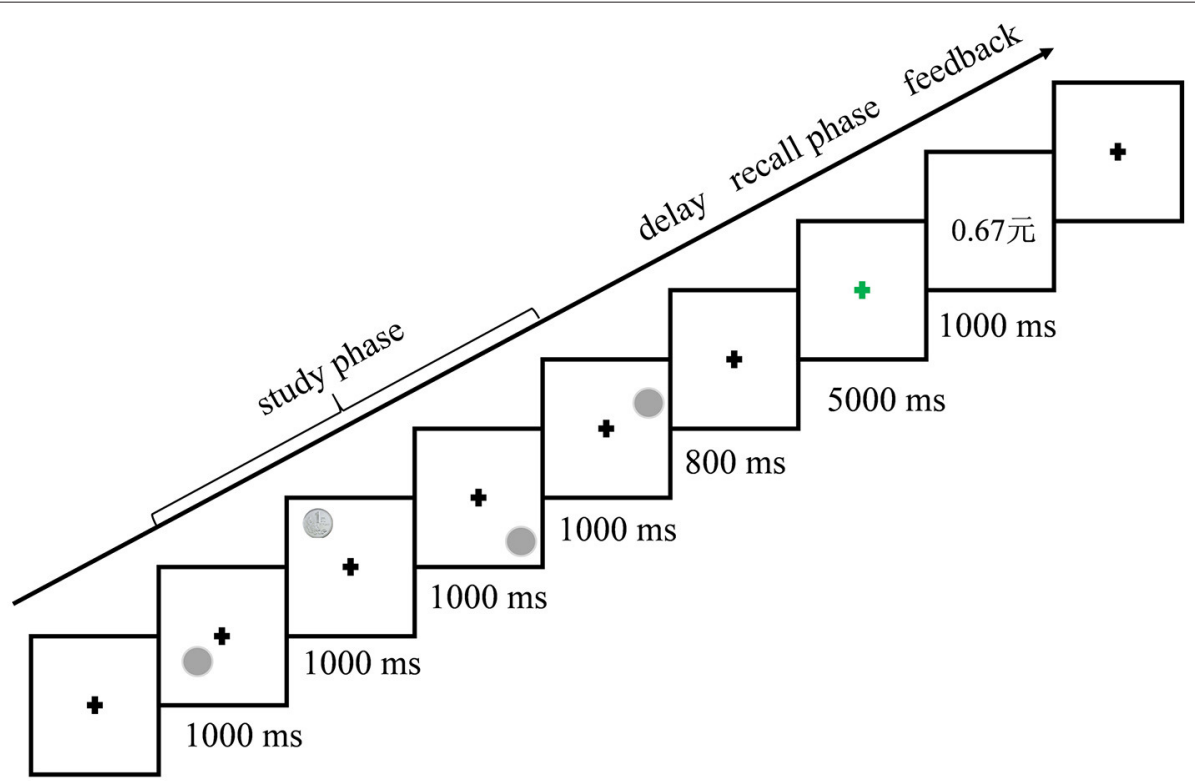

fixating steadily for $800 \mathrm{~ms}$

FIGURE 2 | An example of the sequence of events and presentation times.

$(355 \pm 13) \mathrm{ms} ; M_{\text {High-reward }}=(410 \pm 17) \mathrm{ms} ; F_{(2,38)}=25.736$, $\left.p<0.001, \eta_{\mathrm{p}}^{2}=0.575\right]$, fixation position $\left[M_{\text {cue }}=(421 \pm 17) \mathrm{ms}\right.$; $\left.M_{\text {disk }}=(331 \pm 11) \mathrm{ms} ; F_{(1,19)}=91.351, p<0.001, \eta_{\mathrm{p}}^{2}=0.828\right]$, and their interaction $\left[F_{(2,38)}=15.087, p<0.001, \eta_{\mathrm{p}}^{2}=0.443\right]$. Simple effect analysis showed significant differences in fixation duration between the fixations on locations of reward cue and those on locations of non-cue disk under No-reward $\left[t_{(19)}=\right.$ 8.714, $p<0.001$, Cohen's $d=1.949]$, Low-reward $\left[t_{(19)}=4.810\right.$, $p<0.001$, Cohen's $d=1.075]$, and High-reward expectation conditions $\left[t_{(19)}=7.005, p<0.001\right.$, Cohen's $\left.d=1.566\right]$. Reward expectation effects were significant for fixation durations of both reward cue $\left[F_{(2,38)}=6.020, p<0.001, \eta_{\mathrm{p}}^{2}=0.241\right]$ and non-cue disks $\left[F_{(2,38)}=23.120, p<0.001, \eta_{\mathrm{p}}^{2}=0.549\right]$. For the non-cue disks, fixation durations of High-reward cue were significantly longer than those of Low- $\left[t_{(19)}=2.174, p=0.043\right.$, Cohen's $d=$ $0.486]$ and No-reward cues $\left[t_{(19)}=4.077, p<0.001\right.$, Cohen's $d=$ 
A

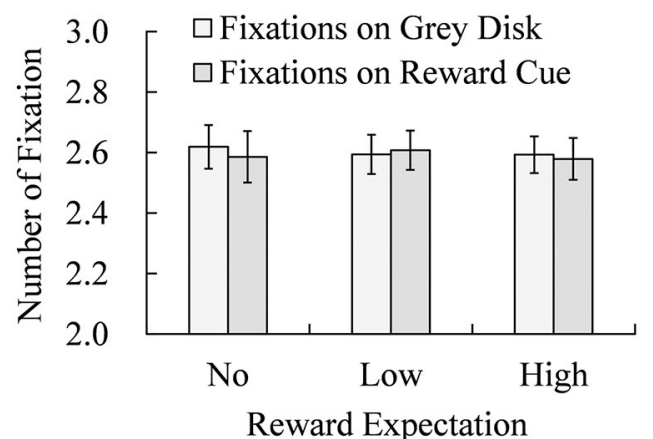

B

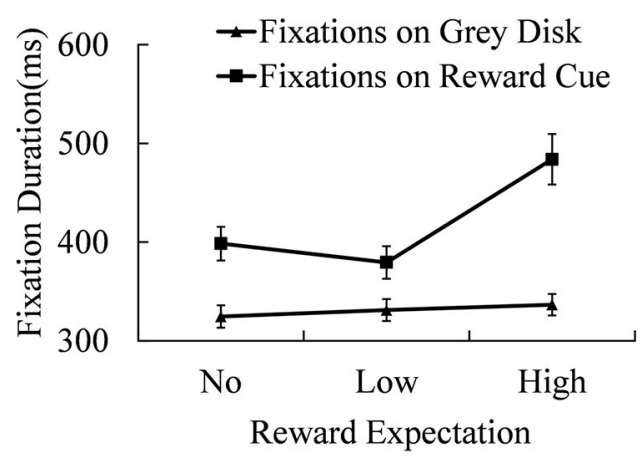

FIGURE 3 | The numbers of fixation (A) and mean fixation duration (B) on cue and non-cue under different reward expectation conditions. Error bars indicate the standard error of mean (SEM).

0.912]. The difference in fixation durations between No-reward and Low-reward cues was marginally significant $\left[t_{(19)}=2.057, p\right.$ $=0.054$, Cohen's $d=0.460$ ]. When reward cues were fixated, the same pattern appeared. Fixation durations of High-reward cues were significantly greater than those of No-reward $\left[t_{(19)}=4.266\right.$, $p<0.001$, Cohen's $d=0.954]$ and Low-reward cues $\left[t_{(19)}=\right.$ $5.596, p<0.001$, Cohen's $d=1.251$ ] , and there was no significant difference between No- and Low-reward cues.

\section{Eye Fixations on the Location of Reward Cue During the Retrieval Phase}

During the retrieval phase, the percentage of cued grids that were correctly fixated (as shown in Figure 4A) and the fixation durations of fixated or unfixated cued grids under different reward expectation conditions were calculated (as shown in Figure 4B).

Analysis of variance (ANOVA) of the percentage of trials in which cued grids were fixated (as shown in Figure 4A) revealed a significant effect of reward expectation $\left[M_{\mathrm{No}-\text { reward }}=51.70 \% \pm\right.$ $1.60 \% ; M_{\text {Low-reward }}=46.40 \% \pm 1.40 \% ; M_{\text {High }- \text { reward }}=29.20 \%$ $\left.\pm 2.50 \% ; F_{(2,38)}=44.800, p<0.001, \eta_{p}^{2}=0.702\right]$. Multiple comparisons showed that the No-reward cued grid was fixated more frequently than the Low-reward $\left[t_{(19)}=2.731, p<0.05\right.$, Cohen's $d=0.886]$ and High-reward cued grids $\left[t_{(19)}=9.461\right.$, $p<0.001$, Cohen's $d=3.070$ ], while the Low-reward cued grid received more fixations than the High-reward cued grid $\left[t_{(19)}=\right.$ 5.702, $p<0.001$, Cohen's $d=1.850]$.

A 2 (fixation on cued grid and non-cued grid) $\times 3$ (No-, Low-, and High-reward cue) repeated-measures ANOVA of fixation duration revealed a significant effect of fixation location $\left[M_{\text {cued }}\right.$ $=(441 \pm 22) \mathrm{ms} ; M_{\text {uncued }}=(461 \pm 19) \mathrm{ms} ; F_{(1,19)}=6.798, p$ $\left.=0.017, \eta_{\mathrm{p}}^{2}=0.263\right]$, reward expectation $\left[M_{\mathrm{No}-\text { reward }}=(470 \pm\right.$ 19) $\mathrm{ms} ; M_{\text {Low-reward }}=(442 \pm 21) \mathrm{ms} ; M_{\text {High-reward }}=(441 \pm 23)$ $\left.\mathrm{ms} ; F_{(2,38)}=5.808, p=0.019, \eta_{\mathrm{p}}^{2}=0.234\right]$, and their interaction $\left[F_{(2,38)}=22.793, p<0.001, \eta_{p}^{2}=0.545\right]$. Simple effect analysis showed significant differences in fixation durations between cued and non-cued grids under No- $\left[F_{(1,19)}=33.280, p<0.001\right.$, $\left.\eta_{\mathrm{p}}^{2}=0.637\right]$, Low- $\left[F_{(1,19)}=23.640, p<0.001, \eta_{\mathrm{p}}^{2}=0.554\right]$, and
High-reward expectation conditions $\left[F_{(1,19)}=11.910, p<0.001\right.$, $\left.\eta_{\mathrm{p}}^{2}=0.385\right]$. Reward expectation effects were significant for mean fixation durations on both cued grid $\left[F_{(2,38)}=11.710, p<0.010\right.$, $\left.\eta_{\mathrm{p}}^{2}=0.381\right]$ and non-cued grid $\left[F_{(2,38)}=15.11, p<0.001\right.$, $\left.\eta_{\mathrm{p}}^{2}=0.443\right]$. Duration of fixation on non-cued grid of No-reward expectation was significantly shorter than that of Low-reward $\left[t_{(19)}=-5.051, p<0.001\right.$, Cohen's $\left.d=1.639\right]$ or High-reward expectation conditions $\left[t_{(19)}=-3.717, p=0.001\right.$, Cohen's $d=$ 1.206]. There was no significant difference in fixation duration between High- and Low-reward expectations. For the cued grid, the fixation duration of No-reward expectation was significantly longer than that of High- $\left[t_{(19)}=6.715, p<0.001\right.$, Cohen's $d=$ 2.179] and Low-reward expectation conditions $\left[t_{(19)}=3.825, p=\right.$ 0.001 , Cohen's $d=1.241$ ], and there was no significant difference between High- and Low- reward expectation conditions.

\section{The Retrieval Performance of Spatial Working Memory Task}

Spatial working memory performances (both GS and LS between the sequence of stimuli positions and eye fixation positions in the recall phase) under different conditions are shown in Figure 5.

For the GS, a 2 (cued grid fixated and unfixated) $\times 3$ (No-, Low-, and High-reward cue) repeated-measures ANOVA revealed a significant effect of reward expectation $\left[M_{\text {No-reward }}\right.$ $=0.601 \pm 0.012 ; M_{\text {Low-reward }}=0.601 \pm 0.010 ; M_{\text {High-reward }}$ $\left.=0.625 \pm 0.011 ; F_{(2,38)}=7.078, p=0.002, \eta_{\mathrm{p}}^{2}=0.271\right]$. The interaction between the cued grid and reward expectation is also significant $\left[F_{(2,38)}=4.356, p=0.020, \eta_{\mathrm{p}}^{2}=0.187\right]$. Simple effect analysis revealed a significant reward expectation effect when the locations of reward were successfully re-fixated $\left[F_{(2,38)}=12.095\right.$, $p<0.001, \eta_{\mathrm{p}}^{2}=0.389$ ] but not when the locations of reward cue were not re-fixated. Further analysis showed that, when the locations of reward cue were successfully re-fixated, there were significant differences in GS between the Low-reward condition and the High-reward condition $\left[t_{(19)}=3.788, p=0.002\right.$, Cohen's $d=0.847$ ] and between the No-reward condition and the Highreward condition $\left[t_{(19)}=4.611, p<0.001\right.$, Cohen's $\left.d=1.031\right]$. However, there was no significant difference between the Noreward condition and the Low-reward condition. The difference 

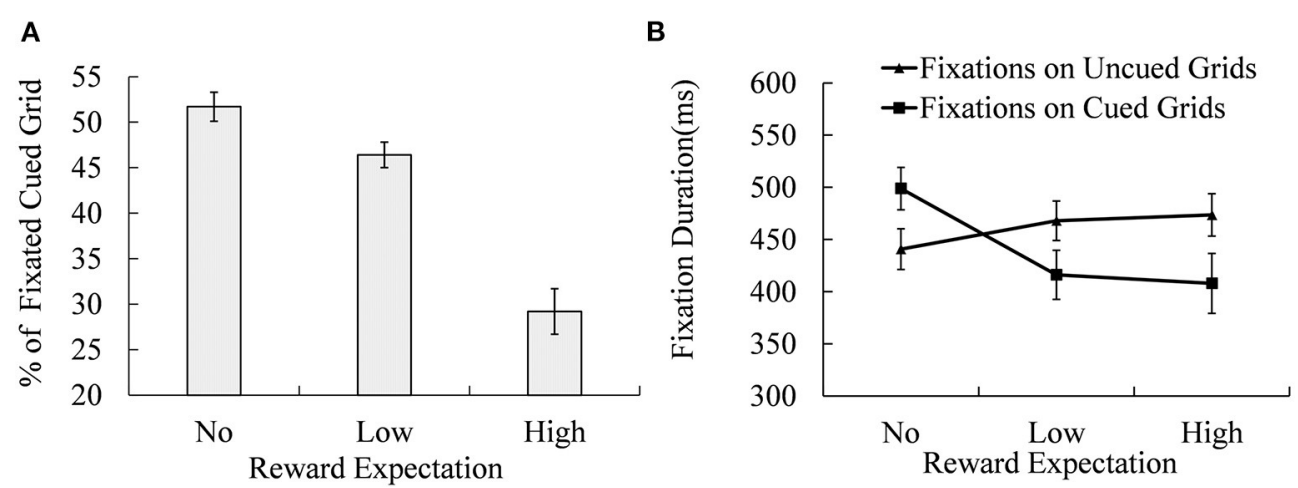

FIGURE 4 | Percentage of the fixated cued locations (A) and the fixation durations of both the cued grids were fixated and unfixated (B) under different reward expectation conditions. Error bars indicate the standard error of mean (SEM).
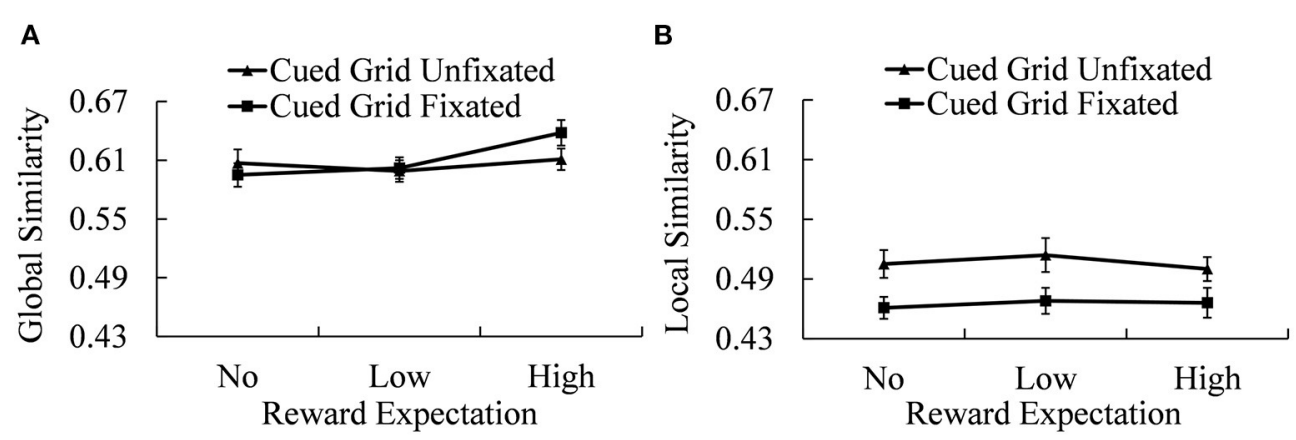

FIGURE 5 | Recall scores of GS (A) and LS (B) under different reward expectation conditions. Error bars indicate the standard error of mean (SEM).

of GS between conditions when the cued grid was fixated and when the cued grid was unfixated was significant in High-reward expectation condition $\left[t_{(19)}=2.813, p=0.011\right.$, Cohen's $d=$ $0.629]$, but not in the other two reward expectation conditions $(p>0.100)$.

Local similarity showed a different statistical pattern, that is, 2 (cued grid fixated and unfixated) $\times 3$ (No-, Low-, and Highreward cue) repeated-measures ANOVA revealed a significant main effect of re-fixation $\left[M_{\text {unfixated }}=0.521 \pm 0.012 ; M_{\text {fixated }}\right.$ $\left.=0.465 \pm 0.010 ; F_{(2,38)}=26.413, p<0.001, \eta_{\mathrm{p}}^{2}=0.582\right]$, showing that when the cued grids failed to be re-fixated, scores of local similarity went up. Other effects on LS were not significant.

\section{DISCUSSION}

The results of the current research showed that reward expectation affected the encoding and maintenance of temporally organized spatial representations. Fourlocation temporal sequence was encoded into the working memory, and more attention was paid to locations with high rewards in the study phase. These spatial location representations were maintained in the delay phase and retrieved successively in the reconstruction phase.

\section{Reward Expectation Enhances Voluntary Attention in the Encoding Phase}

The results of the study phase showed that stimuli, reward cues or not, in the High-reward expectation condition were fixated longer than those in No- and Low-reward expectation conditions (as shown in Figure 5). Considering the effect of reward expectation on recall performance in this study and the indecisive relationship between pure fixation duration and working memory performance in previous studies (Saint-Aubin et al., 2007; Oi et al., 2015), the changed fixation duration in the study phase of this study likely reflect differences in attention allocation under different reward expectation conditions. Specifically, the participants paid more attention to the task when reward expectation was high, compared with the No-reward and Low-reward conditions.

The result of fixation duration in the study phase is in line with previous studies that reported that reward expectation prompted encoding of working memory (Wallis et al., 2015; for a review, see Botvinick and Braver, 2015; Klink et al., 2017; Roberts et al., 2017). In their first experiment, Wallis 
et al. (2015) found that reward improved encoding of rewardassociated items, and the reward effect was generalized to other items in the memory list. In this research, fixation durations on gray disks were prolonged in High-reward conditions as well. It seems that the mechanism underlying the reward effect in the encoding phase is more general than the specified encoding of the reward cue. A plausible explanation is that reward expectation enhances arousal, which in turn provides more cognitive resources for working memory encoding (Murray, 2007; Murayama and Kitagami, 2014; Unsworth and Robison, 2015), and participants are willing to make more efforts to obtain a higher reward. The null result of fixation number and the longer fixation duration of reward cue in the study phase are predictable. The design of the task in this research restricted the saccadic patterns of participants in the study phase and led to equivalent fixations under all reward expectation levels. These reward cues contained additional reward information compared with gray disks, requiring additional processes.

\section{Reward Effects on Different Processing Levels}

The main aim of this research was to explore the reward effect on spatial working memory at different processing levels. As predicted, we found a significant reward effect on GS, the indicator of global processing, but no reward effect on local similarity, the indicator of local processing. Furthermore, the percentage of trials in which the cued grid was correctly re-fixated during the retrieval phase, a relatively local indicator, decreased with the increment of reward. High reward improved spatial working memory performance at the global level but undermined the precision of spatial representations at the local level.

Mean fixation duration in the recall phase is in line with this conclusion. The mean fixation duration in memory-guided saccade tasks is affected by the strength of the memory trace, and it requires more time and effort to recall a weak memory trace (Burke et al., 2012; Haj et al., 2017; Dang et al., 2021). Therefore, when the memory of the next saccade target is weak, prolonged fixation duration is required to generate the following saccade (Meghanathan et al., 2020). Eye-tracking data of this research showed that fixation on uncued locations in the recall phase, sometimes followed by a saccade to the cued location, prolonged as the reward expectation was higher. A potential explanation is that, in this research, the memory for the cued location was degraded when the reward expectation was high, and more time was required before the saccade to the cued location could be generated.

The results of similarities at different processing levels and the negative reward effect on the representation of cued location are consistent with the hypothesis made by Ahmed and Fockert (2012), that is, the authors suggested that working memory load modulates selective attention to different levels of the same stimulus. When working memory load is high, information at a more global level is easily selected, while local-level information is ignored. It is difficult to constrain attention to the local level with a high working memory load. As a result, reward expectation prompted global similarity but did not affect local similarity in this study.

\section{Modulation of Sustained Control on Spatial Working Memory Representation}

An interesting finding of this research is the trade-off between different indicators. As mentioned above, the mean fixation duration of the recall phase reflects degraded representations of cued locations and promoted representations of uncued locations with the increment of reward. Local similarities of trials in which the cued location was falsely re-fixated were higher than local similarities of trials in which the cued location was successfully re-fixated. It seems that the improvement of representations of uncued locations and local similarities comes at the cost of representation of cued locations.

These results of cued-location memory are consistent with the proposal of flexible attention theory (Sandry et al., 2014; Sandry and Ricker, 2020), which suggests that the cognitive system assigns attention resources flexibly among items in working memory, and the elevation of working memory performance of a certain item is at the cost of performance of other items (Sandry et al., 2014; Allen and Ueno, 2018; Sandry and Ricker, 2020). In this research, to obtain a higher reward, goal-directed cognitive control may have inhibited the maintenance of reward-cue location and allocated saved resources to the maintenance of other locations in the delay phase. Moreover, higher local similarities were observed in the trials in which the cued location was falsely re-fixated.

\section{Limitations of This Study}

During the study phase of this study, all three conditions (No-, Low-, and High-) of the reward cue were randomly presented at different temporal positions. It may lead to a confounding effect. The different intervals of processing the reward cue mean that the levels of processing or the motivational states may vary accordingly. Specifically, motivational states might be identical in the three reward conditions until the presentation of the reward cue. Moreover, according to Sandry and Ricker (2020), the temporal position does affect performance in working memory tasks. Hence, the temporal position of reward cues should be considered in future studies.

\section{CONCLUSION}

By applying sequential memory-guided eye movement tasks, we reached the following conclusions regarding the reward effect on spatial working memory: (a) reward expectation enhances the encoding of spatial locations by improving voluntary attention, and (b) reward affects reconstruction only at the global level but not at the local level, in which the cognitive resource is reallocated among reward- and nonreward-associated items in working memory to maximize the reward effect. 


\section{DATA AVAILABILITY STATEMENT}

The raw data supporting the conclusions of this article will be made available by the authors, without undue reservation.

\section{ETHICS STATEMENT}

The studies involving human participants were reviewed and approved by Psychological Ethics Committee of Capital Normal University. The patients/participants

\section{REFERENCES}

Ahmed, L., and Fockert, J. W. (2012). Working memory load can both improve and impair selective attention: evidence from the navon paradigm. Attent. Percept. Psychophys. 74, 1397-1405. doi: 10.3758/s13414-012-0357-1

Allen, R. J., and Ueno, T. (2018). Multiple high-reward items can be prioritized in working memory but with greater vulnerability to interference. Attent. Percept. Psychophys. 80, 1-13. doi: 10.3758/s13414-018-1543-6

Anna, H., and Anna, S. (2018). Separate and combined effects of action relevance and motivational value on visual working memory. J. Vis. 18, 1-14. doi: $10.1167 / 18.5 .14$

Antoine, S., Ranzini, M., van Dijck, J., Slama, H., Bonato, M., and Tousch, A., et al. (2018). Hemispatial neglect and serial order in verbal working memory. J. Neuropsychol. 13, 272-288. doi: 10.1111/jnp. 12145

Barrett, L. F., Tugade, M. M., and Engle, R. W. (2004). Individual differences in working memory capacity and dual-process theories of the mind. Psychol. Bullet. 130, 553-573. doi: 10.1037/0033-2909.130.4.553

Beck, S. M., Locke, H. S., Savine, A. C., Jimura, K., and Braver, T. S. (2010). Primary and secondary rewards differentially modulate neural activity dynamics during working memory. PLoS ONE 5:e9251. doi: 10.1371/journal.pone.0009251

Botvinick, M. M., and Braver, T. (2015). Motivation and cognitive control: from behavior to neural mechanism. Ann. Rev. Psychol. 66, 83-113. doi: 10.1146/annurev-psych-010814-015044

Brandt, S. A., and Stark, L. W. (1997). Spontaneous eye movements during visual imagery reflect the content of the visual scene. J. Cogn. Neurosci. 9, 27-38. doi: 10.1162/jocn.1997.9.1.27

Burke, M. R., Allen, R. J., and Gonzalez, C. (2012). Eye and hand movements during reconstruction of spatial memory. Perception 41, 803-818. doi: $10.1068 / \mathrm{p} 7216$

Cho, Y. T., Lam, N. H., Starc, M., Santamauro, N., Savic, A., and Diehl, C. K., et al. (2018). Effects of reward on spatial working memory in schizophrenia. J. Abnorm. Psychol. 127, 695-709. doi: 10.1037/abn0000369

Choi, J. M., Padmala, S., and Pessoa, L. (2015). Counteracting effect of threat on reward enhancements during working memory. Cogn. Emot. 29, 1517-1526. doi: 10.1080/02699931.2014.993596

Cowan, N. (2001). The magical number 4 in short-term memory: a reconsideration of mental storage capacity. Behav. Brain Sci. 24, 87-185. doi: $10.1017 / \mathrm{S} 0140525 \mathrm{X} 01003922$

Dang, X. X., Li, L. F., Chen, Y. H., and Yang, X. J. (2021). Distinguishing between high-confidence true and false memories: evidence from eye movements. Austr. J. Psychol. 11, 1-12. doi: 10.1080/00049530.2020.1865779

Di Rosa, E., Brigadoi, S., Cutini, S., Tarantino, V., Dell'Acqua, and, R., Mapelli, D., et al. (2019). Reward motivation and neurostimulation interact to improve working memory performance in healthy older adults: a simultaneous tdcsfNIRS study. NeuroImage 202:116062. doi: 10.1016/j.neuroimage.2019.116062

Eddy, S. R. (2004). What is dynamic programming? Nat. Biotechnol. 22, 909-910. doi: 10.1038/nbt0704-909

Esteban, L., Vivas, A. B., Fuentes, L. J., and Estévez, A. F. (2015). Spatial working memory is enhanced in children by differential outcomes. Sci. Rep. 5:17112. doi: $10.1038 /$ srep 17112

Fairclough, S. H., Burns, C., and Kreplin, U. (2018). Fnirs activity in the prefrontal cortex and motivational intensity: impact of working memory load, financial provided their written informed consent to participate in this study.

\section{AUTHOR CONTRIBUTIONS}

JD and QZ discussed and developed the idea and wrote the introduction, methods, results, and discussion. ZJ designed the study with input from JD and QZ and ran the experiment on all participants. JD and ZJ were highly involved in the analysis of the results. All authors contributed to the article and approved the submitted version.

reward, and correlation-based signal improvement. Neurophotonics 5:035001. doi: 10.1117/1.NPh.5.3.035001

Fairclough, S. H., and Ewing, K. (2017). The effect of task demand and incentive on neurophysiological and cardiovascular markers of effort. Int. J. Psychophysiol. 119, 58-66. doi: 10.1016/j.ijpsycho.2017.01.007

Filetti, M., Barral, O., Jacucci, G., and Ravaja, N. (2019). Motivational intensity and visual word search: layout matters. PLoS ONE 14:e0218926. doi: 10.1371/journal.pone.0218926

Fischer-Baum, S., and Benjamin, A. S. (2014). Time, space, and memory for order. Psychon. Bullet. Rev. 21, 1263-1271. doi: 10.3758/s13423-014-0 604-7

Friedman, A., and Liebelt, L. S. (1981). On the time course of viewing pictures with a view towards remembering. Eye Mov. Cogn. Vis. Percept. 12, 137-155. doi: 10.4324/9781315437415-12

Funahashi, S., Bruce, C. J., and Goldmanrakic, P. S. (1993). Dorsolateral prefrontal lesions and oculomotor delayed-response performance: evidence for mnemonic "scotomas". J. Neurosci. 13, 1479-1497. doi: 10.1523/JNEUROSCI.13-04-01479.1993

Gaillard, C., Guillod, M., Ernst, M., Torrisi, S., Federspiel, A., and Schoebi, D., et al. (2019). Striatal responsiveness to reward under threat of shock and working memory load: a preliminary study. Brain Behav. 9:1397. doi: 10.1002/brb3.1397

Gilbert, A. M., and Fiez, J. A. (2004). Integrating rewards and cognition in the frontal cortex. Cogn. Affect. Behav. Neurosci. 4, 540-552. doi: $10.3758 / \mathrm{CABN} .4 .4 .540$

Hager, O. M., Dirschner, M., Bischof, M., Hartmann-Riemer, M. N., Kluge, A., and Seifritz, E., et al. (2015). Reward-dependent modulation of working memory is associated with negative symptoms in schizophrenia. Schizophr. Res. 168, 238-244. doi: 10.1016/j.schres.2015.08.024

Haj, M. E., Nandrino, J. L., Antoine, P., Boucart, M., and Lenoble, Q. (2017). Eye movement during retrieval of emotional autobiographical memories. Acta Psychol. 174, 54-58. actpsy.2017.02.002 doi: 10.1016/j.actpsy.2017.02.002

Hammer, R., Tennekoon, M., Cooke, G. E., Gayda, J., Stein, M. A., and Booth, J. R. (2015). Feedback associated with expectation for larger-reward improves visuospatial working memory performances in children with ADHD. Dev. Cogn. Neurosci. 14, 38-49. doi: 10.1016/j.den.2015.06.002

Hefer, C., and Dreisbach, G. (2017). How performance-contingent reward prospect modulates cognitive control: increased cue maintenance at the cost of decreased flexibility. J. Exp. Psychol. Learn. Mem. Cogn. 43, 1643-1658. doi: $10.1037 / x \operatorname{lm} 0000397$

Heitz, R. P., Schrock, J. C., Payne, T. W., and Engle, R. W. (2008). Effects of incentive on working memory capacity: behavioral and pupillometric data. Psychophysiology 45, 119-129. doi: 10.1111/j.1469-8986.2007.00605.x

Heritage, A. J., Long, L. J., Woodman, G. F., and Zald, D. H. (2017). Personality correlates of individual differences in the recruitment of cognitive mechanisms when rewards are at stake. Psychophysiology 55:12987. doi: 10.1111/psyp.12987

Hopstaken, J. F., Dimitri, V., Bakker, A. B., Kompier, M., and Leung, Y. K. (2016). Shifts in attention during mental fatigue: evidence from subjective, behavioral, physiological, and eye-tracking data. J. Exp. Psychol. Hum. Percept. Perform. 42, 878-889. doi: 10.1037/xhp0000189

Infanti, E., Hickey, C., Menghi, N., and Turatto, M. (2017). Reward-priming impacts visual working memory maintenance: evidence from human electrophysiology. Vis. Cogn. 25:1365790. doi: 10.1080/13506285.2017.1365790 
Johnston, K., and Everling, S. (2008). Neurophysiology and neuroanatomy of reflexive and voluntary saccades in non-human primates. Brain Cogn. 68, 271-283. doi: 10.1016/j.bandc.2008.08.017

Just, M. A., and Carpenter, P. A. (1992). A capacity theory of comprehension: individual differences in working memory. Psychol. Rev. 99, 122-149. doi: 10.1037/0033-295X.99.1.122

Kawasaki, M., and Yamaguchi, Y. (2013). Frontal theta and beta synchronizations for monetary reward increase visual working memory capacity. Soc. Cogn. Affect. Neurosci. 5, 523-530. doi: 10.1093/scan/nss027

Klink, P. C., Jeurissen, D., Theeuwes, J., Denys, D., and Roelfsema, P. R. (2017). Working memory accuracy for multiple targets is driven by reward expectation and stimulus contrast with different time-courses. Sci. Rep. 7:9082. doi: 10.1038/s41598-017-08608-4

Krawczyk, D. C., Gazzaley, A., and D’Esposito, M. (2007). Reward modulation of prefrontal and visual association cortex during an incentive working memory task. Brain Res. 1141, 168-177. doi: 10.1016/j.brainres.2007.01.052

Lavie, N., Hirst, A., de Fockert, J. W., and Viding, E. (2004). Load theory of selective attention and cognitive control. J. Exp. Psychol. Gen. 133, 339-354. doi: 10.1037/0096-3445.133.3.339

Ludwig, C. J. H., Davies, J. R., and Eckstein, M. P. (2014). Foveal analysis and peripheral selection during active visual sampling. Proc. Natl. Acad. Sci. U. S. A. 111, 291-299. doi: 10.1073/pnas.1313553111

Magis-Weinberg, L., Custers, R., and Dumontheil, I. (2019). Rewards enhance proactive and reactive control in adolescence and adulthood. Soc. Cogn. Affect. Neurosci. 14, 1-14. doi: 10.1093/scan/nsz093

Manga, A., Vakli, P., and Vidnyánszky, Z. (2020). The influence of anticipated monetary incentives on visual working memory performance in healthy younger and older adults. Sci. Rep. 10:8817. doi: 10.1038/s41598-020-65723-5

Marchette, S. A., Sever, M. W., Flombaum, J. I., and Shelton, A. L. (2015). Individual differences in representational precision predict spatial working memory span. Spat. Cogn. Comput. Interdiscipl. J. 15, 308-328. doi: 10.1080/13875868.2015.1078334

Marquand, A. F., De Simoni, S., O’Daly, O. G., Williams, S. C. R., MouralMiranda, J., and Mehta, M. A. (2011). Pattern classification of working memory networks reveals differential effects of methylphenidate, atomoxetine, and placebo in healthy volunteers. Neuropsychopharmacology 36, 1237-1247. doi: $10.1038 / \mathrm{npp} .2011 .9$

Meghanathan, R. N., Leeuwen, C. V., Giannini, M., and Nikolaev, A. R. (2020). Neural correlates of task-related refixation behavior. Vis. Res. 175, 90-101. doi: 10.1016/j.visres.2020.07.001

Miller, G. A. (1956). The magical number seven plus or minus two: some limits on our capacity for processing information. Psychol. Rev. 63, 81-97. doi: $10.1037 / \mathrm{h} 0043158$

Murayama, K., and Kitagami, S. (2014). Consolidation power of extrinsic rewards: reward cues enhance long-term memory for irrelevant past events. J. Exp. Psychol. 143, 15-20. doi: 10.1037/a0031992

Murray, E. A. (2007). The amygdala, reward and emotion. Trends Cognitiv. 11, 489-497. doi: 10.1016/j.tics.2007.08.013

Oi, Y., Ikeda, Y., Okuzumi, H., Kokubun, M., Hamada, T., and Sawa, T. (2015). Fixation effects on forward and backward recall in a spatial working memory task. Psychology 6, 727-733. doi: 10.4236/psych.2015.66071

Papageorgiou, K. A., Smith, T. J., Wu, R., Johnson, M. H., Kirkham, N. Z., and Ronald, A. (2014). Individual differences in infant fixation duration relate to attention and behavioral control in childhood. Psychol. Sci. 25, 1371-1379. doi: $10.1177 / 0956797614531295$

Park, S., Holzman, P. S., and Goldman-Rakic, P. S. (1995). Spatial working memory deficits in the relatives of schizophrenic patients. Archiv. Gen. Psychiatr. 52, 821-828. doi: 10.1001/archpsyc.1995.03950220031007

Pessoa, L. (2009). How do emotion and motivation direct executive control? Trends Cogn. Sci. 13, 160-166. doi: 10.1016/j.tics.2009.01.006

Pochon, J. B., Levy, R., Fossati, P., Lehericy, S., Poline, J. B., et al. (2002). The neural system that bridges rewards and cognition in humans: an fMRI study. Proc. Natl. Acad. Sci. U. S. A. 99, 5669-5674. doi: 10.1073/pnas.082111099

Qu, L., Finestone, D. L., Qin, L. J., and Reena, Z. X. (2013). Focused but fixed: the impact of expectation of external rewards on inhibitory control and flexibility in preschoolers. Emotion 13, 562-572. doi: 10.1037/a0027263

Rayner, K. (1978). Eye movements in reading and information processing. Psychol. Bullet. 85, 618-660. doi: 10.1037/0033-2909.85.3.618
Rayner, K. (1998). Eye movements in reading and information processing: 20 years of research. Psychol. Bullet. 124, 372-422. doi: 10.1037/0033-2909.124.3.372

Rayner, K. (2009). Eye movements and attention in reading, scene perception, and visual search. Quart. J. Exp. Psychol. 62, 1457-1506. doi: 10.1080/17470210902816461

Rayner, K., Li, X., Williams, C. C., Cave, K. R., and Well, A. D. (2007). Eye movements during information processing tasks: individual differences and cultural effects. Vis. Res. 47, 2714-2726. doi: 10.1016/j.visres.2007. 05.007

Roberts, W. A., Macdonald, H., Brown, L., and Macpherson, K. (2017). Release from proactive interference in rat spatial working memory. Learn. Behav. 45, 263-275. doi: 10.3758/s13420-017-0263-4

Rowe, J. B., Eckstein, D., Braver, T., and Owen, A. M. (2008). How does reward expectation influence cognition in the human brain? J. Cogn. Neurosci. 20, 1980-1992. doi: 10.1162/jocn.2008.20140

Saarinen, J. (1988). Perception of spatial order in extrafoveal vision. J. Psychol. 29, 162-167. doi: 10.1111/j.1467-9450.1988.tb00787.x

Saint-Aubin, J., Tremblay, S., and Jalbert, A. (2007). Eye movements and serial memory for visual-spatial information: does time spent fixating contribute to recall? Exp. Psychol. 54, 264-272. doi: 10.1027/1618-3169.54. 4.264

Sandry, J., and Ricker, T. J. (2020). Prioritization within visual working memory reflects a flexible focus of attention. Attent. Percept. Psychophys. 82, 2985-3004. doi: 10.3758/s13414-020-02049-4

Sandry, J., Schwark, J. D., and Macdonald, J. (2014). Flexibility within working memory and the focus of attention for sequential verbal information does not depend on active maintenance. Mem. Cogn. 42, 1130-1142. doi: 10.3758/s13421-014-0422-1

Savine, A. C., Beck, S. M., Edwards, B. G., Chiew, K. S., and Braver, T. S. (2010). Enhancement of cognitive control by approach and avoidance motivational states. Cogn. Emot. 24, 338-356. doi: 10.1080/02699930903381564

Sawaguchi, T., and Iba, M. (2001). Prefrontal cortical representation of visuospatial working memory in monkeys examined by local inactivation with muscimol. $J$. Neurophysiol. 86, 2041-2053. doi: 10.1152/jn.2001.86.4.2041

Smith, A. E., Dalecki, S. J., and Crystal, J. D. (2017). A test of the reward-value hypothesis. Anim. Cogn. 20, 215-220. doi: 10.1007/s10071-016-1040-z

Su, Z., Wang, L., Kang, G., and Zhou, X. (2021). Reward makes the rhythmic sampling of spatial attention emerge earlier. Attent. Percept. Psychophys. 83:5. doi: 10.3758/s13414-020-02226-5

Taylor, S. F., Welsh, R. C., Wager, T. D., Phan, K. L., Fitzgerald, K. D., and Gehring, W. J. (2004). A functional neuroimaging study of motivation and executive function. Neuroimage 21, 1045-1054. doi: 10.1016/j.neuroimage.2003. 10.032

Theeuwes, J., Belopolsky, A. V., and Olivers, C. N. L. (2009). Interactions between working memory, attention, and eye movements. Acta Psychol. 132, 106-114. doi: 10.1016/j.actpsy.2009.01.005

Thurm, F., Zink, N., and Li, S. C. (2018). Comparing effects of reward anticipation on working memory in younger and older adults. Front. Psychol. 9:2318. doi: 10.3389/fpsyg.2018.02318

Tsujimoto, S., and Postle, B. R. (2012). The prefrontal cortex and oculomotor delayed response: a reconsideration of the "mnemonic scotoma". J. Cogn. Neurosci. 24, 627-635. doi: 10.1162/jocn_a_00171

Unsworth, N., and Robison, M. K. (2015). Individual differences in the allocation of attention to items in working memory: evidence from pupillometry. Psychon. Bullet. Rev. 22, 757-765. doi: 10.3758/s13423-0140747-6

van Dijck, J. P., Abrahamse, E. L., Majerus, S., and Fias, W. (2013). Spatial attention interacts with serial-order retrieval from verbal working memory. Psychol. Sci. 24, 1854-1859. doi: 10.1177/0956797613479610

Vergilino, D., and Beauvillain, C. (2001). Reference frames in reading: evidence from visually and memory-guided saccades. Vis. Res. 41, 3547-3557. doi: 10.1016/S0042-6989(01)00225-5

Wallis, G., Stokes, M. G., Arnold, C., and Nobre, A. C. (2015). Reward boosts working memory encoding over a brief temporal window. Vis. Cogn. 23, 291-312. doi: 10.1080/13506285.2015.1013168

Wei, P., and Kang, G. (2014). Task relevance regulates the interaction between reward expectation and emotion. Exp. Brain Res. 232, 1783-1791. doi: $10.1007 / \mathrm{s} 00221-014-3870-8$ 
Conflict of Interest: The authors declare that the research was conducted in the absence of any commercial or financial relationships that could be construed as a potential conflict of interest.

Publisher's Note: All claims expressed in this article are solely those of the authors and do not necessarily represent those of their affiliated organizations, or those of the publisher, the editors and the reviewers. Any product that may be evaluated in this article, or claim that may be made by its manufacturer, is not guaranteed or endorsed by the publisher.

Copyright (c) 2021 Zhou, Jiang and Ding. This is an open-access article distributed under the terms of the Creative Commons Attribution License (CC BY). The use, distribution or reproduction in other forums is permitted, provided the original author(s) and the copyright owner(s) are credited and that the original publication in this journal is cited, in accordance with accepted academic practice. No use, distribution or reproduction is permitted which does not comply with these terms. 